\title{
The role of intelligence in economic decision making
}

Aldo Rustichini, Department of Economics, University of Minnesota

Corresponding author: Aldo Rustichini

Email: aldo.rustichini@gmail.com; telephone: 16126254816 fax: 16126240209

\begin{abstract}
Research on the link between intelligence and economic decision making is a recent development in the more general attempt to introduce theories of individual differences and personality traits into the analysis of economic behavior. We lay down here what we know from behavioral studies, from imaging studies, both functional and anatomical, and insights from decision theory and game theory. All the results point to a correlation and perhaps a deeper link between cognition and decision making, both in single-player and in strategic environments. We see several pieces of a puzzle, and provide some suggestions on how future research will discover the hidden image.
\end{abstract}

\section{Introduction}

An operational way of defining intelligence [1] begins with the empirical observation that test scores on cognitive tasks are positively correlated. If one then looks for an explanation of this regularity through exploratory factor analysis, one finds that scores in specific tests can be explained in a satisfactory way by a general factor (which Spearman called $g$ ) and an independent, task-specific factor. These conclusions have long been controversial, but they seem to be now widely accepted [2]. We will focus here on $g$ (and call it intelligence) as the measurable individual characteristic of performance in general cognitive processes.

Economic decision making is the selection of one from a feasible set of options, each one having a value to the decision maker, and involves the processing of information on several relevant variables describing the options. This process is already complex in the case of an individual acting in isolation, as it requires an understanding of the options offered, whether at the supermarket or in the laboratory, an introspective evaluation of the prospective pleasure derived from each, perhaps on the basis of previous 
experiences, and the risk or time delay involved in the case of monetary payments. Information processing is even more complex in decision making in a strategic environment, where the consequences depends both on the choice made by the individual and the choices made by others. We will examine here the relation between intelligence and economic decision making; we ignore the obviously important, related but different issue of intelligence and economic outcomes (which is discussed instead for instance in $[3,4,5,6,7])$.

\section{Intelligence and the method of choice}

It seems natural to consider decision making as a special cognitive task, provide a definition of performance in this case, and expect (because of the $g$ factor) this performance to be correlated with that exhibited in other tasks. The definition of performance in economic choices should not bind the individual to a particular preference over options, but should only constrain the method of choice. For example, if $\mathbf{a}, \mathbf{b}$, and $\mathbf{c}$ are lotteries with monetary payments, the choice between $\mathbf{a}$ and $\mathbf{b}$ and that between $\mathbf{b}$ and $\mathbf{c}$ should be entirely a matter of taste, and have no bearing on the general cognitive ability of the person who is doing the choosing. However, a reasonable consistency requirement (called transitivity) in choice is that if you prefer option $\mathbf{a}$ to $\mathbf{b}$, and $\mathbf{b}$ to $\mathbf{c}$, then you should not prefer $\mathbf{c}$ to $\mathbf{a}$. That this is the case has been experimentally verified in recent years $([8,9,10,11,12])$ : individuals with higher scores in IQ tests (e.g. Raven matrices) are more likely to be consistent. Similarly, individuals with higher intelligence should be less sensitive to irrelevant details in the presentation of the options (framing).

A similar correlation might also seem reasonable in strategic environments, although in this case the restrictions on behavior are substantially weaker. This is a consequence of the fact that, since the outcome of an action depends on the actions of others, and prediction of what the other will do depends on what they think you will do, different actions might be equally reasonable depending on appropriate beliefs about what the others will do. A solution concept (that is, a theory that selects some joint behavior of players among all the possible ones) exists $[13,14]$ that only requires an action to be justifiable for some belief on what the others are going to do. This is the case if the action is the best choice given some belief about the choice of others, provided those beliefs are in turn a best choice given some belief about the choice of others, and so on. Even if one adopts this criterion (called rationalizability, which is weaker than the usual Nash equilibrium concept), some restrictions on behavior follow. For example, no action $\mathbf{b}$ should be taken that gives a worse outcome than a no matter what the others are doing (that is, no rational player should use a dominated action). There is still no 
systematic experimental test for the relation between intelligence and some appropriate definition of performance in choice, or rationalizability in games, although this is clearly an interesting field for future research.

A substantially more interesting line of research, however, is motivated by the finding that the role of intelligence goes well beyond the positive correlation between performance in choice and in cognitive tasks; instead, it discovers a relation between preferences and intelligence. In this survey we will focus on this second aspect of the relation between intelligence and what preferences are, rather than how they are implemented in choices.

\section{Intelligence and preferences}

A famous example of the relation between intelligence and preferences is in the realm of choice among rewards delivered at different points in time. In psychology, this has been known since Mischel's [15] marshmallow experiment: children's ability to postpone eating a marshmallow now, in exchange for an additional one later, was found to be correlated with performance in SAT tests, and in general with educational and economic success later in life. Some recent research has shown that a similar correlation holds for other domains of choice, in particular choice under risk and uncertainty. For example, we have argued elsewhere that there is no reason why we should consider the choice of a fifty-fifty chance of 100 dollars over 40 dollars more reasonable than the opposite. Nevertheless, just as with preferences over dated rewards, the pattern of choices under risk exhibits a correlation with intelligence. This relation, discussed in $[8,9,10,11,12]$ cited earlier, is complex, but in general a greater willingness to risk is associated with higher intelligence.

A correlation between intelligence and behavior is also found in strategic behavior. This relation has probably deep roots: the social intelligence hypothesis $[16,17,18,19,20]$ suggests that the richness of the social interaction in humans demands the development of flexible cognitive strategies, as opposed to adaptive rules of thumb. A natural way to predict how behavior in a strategic environment correlates with intelligence is to assume that higher intelligence will bring individuals closer to the behavior predicted by game-theoretic equilibrium concepts, based on the assumption of rationality of the players. When the game-theoretic prediction is unique, the restriction is powerful. This prediction has been experimentally tested, and has found some support [21, 22, 23, 24]. However, there are some interesting exceptions to this rule when players do not have opposing interests, and there are potential gains from cooperation. An important example is provided by the sequential two-player trust game: the

first mover has to choose an investment, paid for out of his own funds; the investment gives a return 
which is paid to the second player, who is informed of the amount paid by the first player and has to decide how much to give to the first out of his new total wealth. A rational second player who is only interested in his payoffs will transfer nothing back, and the first player, anticipating that his investment will give no benefit to him, will invest nothing. The assumption suggested earlier that higher intelligence is associated with more rational behavior would lead to the prediction that players with higher intelligence will transfer less. Instead, the opposite holds in experimental tests [9]: higher intelligence players transfer more as first mover and are more reciprocal as second mover. Similar results hold for the one-shot Prisoner's Dilemma [25].

When the game-theoretic prediction is not unique strategic analysis provides little guidance to the relation between intelligence and behavior. A lack of a unique prediction is the rule in repeated games, where two players play the same simultaneous move game for many rounds, for example the Prisoner's Dilemma [26, 27*]. In experimental setups, the probability that the repeated encounters come to an end in each round is decided by a random device; the higher the continuation probability, the higher a consideration of future rounds will weigh on the current decision, and make cooperation today to induce cooperation tomorrow more appealing. Note that the set of possible equilibria is still very large, and since intelligence is not explicitly considered in game theory, the theory is silent on the relation between intelligence and behavior. There are, however, interesting regularities. For instance, [Proto E, Rustichini A, Sofianos A, under review] if players are allocated to two groups of high and low IQ score, then the cooperation rate is very similar across groups in the early stages but diverges substantially over the experimental session, with high IQ score players reaching a cooperation level close to $100 \%$, and low IQ score ones drifting to lower cooperation rates.

\section{Model-free learning and intelligence}

Research on neural correlates into the way intelligence modulates information processing on rewards has so far been confined to model-free learning theories. These theories view learning as adjusting the assessment of the value of an action in proportion to the difference (called prediction error) between the reward obtained and the expectation of the reward according to an earlier assessment. This adjustment does not require an understanding or a modeling of the structure of the transition probability among states of nature that affect rewards: this transition is instead explicitly introduced in model-based theories, which assume that the individuals learn what the transition is.

Even this simple class of models provides some insight into the role of intelligence in learning $[28,29]$. In $\left[30^{*}\right]$ the authors find a positive correlation between the prediction error in the ventral 
striatum (VS) and fluid intelligence. A related result, but in the right dorso-Lateral Prefrontal Cortex (DLPFC) and the dorsal Anterior Cingulate Cortex (dACC) is found in [31*]. A different result (a negative correlation between the direction of the prediction error and IQ) is found in [32*]. The three results may be considered to be broadly consistent if one takes into account a crucial difference in the experimental designs. In [32], the subjects did not know the process governing rewards and they had to learn it. An analysis of their behavior shows that subjects with higher IQ scores are likely to learn faster the fact that they are facing an approximately equal probability of reward independently of their choice. Thus, the common feature of these studies is that in model-free learning, higher intelligence subjects are faster at learning the relevant properties of the environment.

\section{Caudate Volume and Intelligence}

These results indicate a link between the learning properties of the process delivering rewards and intelligence. Several recent studies, some in the clinical literature, have investigated a possible link between individual differences in caudate volume and intelligence [33]. A correlation between caudate volume and IQ score has been found in 105 preadolescent prematurely born children [34]. Similarly, a correlation between Verbal IQ (but not Performance IQ) and caudate volume has been found in 76 adolescents born prematurely [35*]. A study investigating the link between respiratory fitness and cognitive decline found that caudate volume mediated the association between cardiopulmonary fitness and cognitive flexibility in 179 elderly adults [36]. An association between intelligence and the shape of right hemisphere striatal structures (including the caudate, putamen, nucleus accumbens, and thalamus) was found in a sample of 93 healthy adults [37]. A positive association between intelligence and caudate volume has also been found in three large independent samples of healthy adults (total $N=517$ ) [38]; the relation holds for bilateral caudate, after controlling for age, sex and total brain volume, and it is strong for Verbal IQ but weaker for Performance IQ. The effect size is similar in the three sub-samples. Instead, no correlation holds with the volume of other subcortical structures.

\section{Learning and risk taking}

The findings we reported, linking intelligence and structural properties of anatomical regions of the brain are seemingly robust, and have been replicated in different and independent samples. A very interesting direction of research now opens, which is specific to our topic, namely the link between intelligence and properties of economic decision making. Since the regions found to be related to intelligence are also linked to reward processing, a natural first question is whether these results 
provide an insight into the link between intelligence and attitude to risk identified in earlier studies? Building on some simple learning models $[39,40]$ some modeling research illustrates how such a link is possible $[41,42,43]$.

For example, [43] models experimentation in choice as follows: suppose someone is repeatedly choosing between a risky reward and a safe one. From the outcomes observed he has to make an estimate of the value of the risky estimate and has to decide in every period which of the two options to sample. The model makes two simple assumptions. The first is that the current estimate when the risky choice is taken is a weighted average with constant coefficients of the past estimates and the currently observed reward; if the choice is not risky, then the current estimate is set equal to previous. Second, the probability of choosing the risky option is a logistic function of its estimated value. If the expected value of the risky choice is equal to the safe amount, then the expectation of the choice of the risky option is less than fifty per cent (so the decision maker behaves as risk averse). The reason for this is a tendency to avoid sampling when the estimate is low. Thus a negative outcome creates a trap in which the decision maker chooses not to learn the true value. The model is very simple, and the adaptive learning rule may seem arbitrary, but it clearly shows how risk attitudes can be produced by learning. Research in this direction can provide insights linking the behavioral, brain imaging and anatomical results we have seen.

Model-free learning theories are useful, but probably too simple to give us a full understanding of the role of intelligence in decision making and learning. Research is currently under way to evaluate the role of intelligence in a richer class, called model-based theories. These theories model the learning environment as a state variable and a transition among states that may depend on the actions of subjects. A model-based theory makes the state and the transition an explicit component of the conceptual structure used by the decision maker, who may learn about parameters describing the transition as well as the state. Two sub-classes of models-based theories are particularly suitable to investigate the role of intelligence in learning [44]; they differ with respect to what is known about the state, which may be observable [45] or hidden [46]. The latter group seems most relevant to studying the role of intelligence in economic decision making.

\section{How intelligence affects economic decision making: future research}

We have reviewed several findings providing a link between intelligence and economic, including strategic, decision making. Economic decision making is a special task, distinct from the typical cognitive task, because in economic decision making there is no wrong or correct answer. So the 
research we surveyed is an attempt to explain what is specific to this realm of decision making, namely the features of choice pattern, such as attitude to risk or patience in choice over timed rewards, rather than performance, such as the consistency of choice.

The research should now, we think, proceed to look for a unified an understanding of the reasons for the existence of this link. A first step in understanding how intelligence affects economic decision making should probably proceed by first establishing a benchmark for optimal choice considered as information processing. A subject observes a sequence of signals (for example rewards obtained in the past) providing information on payoff-relevant states, which may be only partially observable. The subject formulates a belief on the state and chooses actions taking into account the effect on rewards as well as the informational value of the signal he gets. One can formulate the benchmark model as a dynamic programming problem taking the set of beliefs as state space, and use Bayesian updating, or (in a method closer to model-based theories) adjust one's estimates on the basis of the prediction error. The effectiveness of information processing may be affected by intelligence in several ways: by the quality of the signal observed, by the amount of information retained in working memory, or finally by the quality of inference (for example the noise in the updating processing. The cost function may be taken to model intelligence: a higher cost associated with lower cognitive skill scores. Once this benchmark is established, realistic models of the way in which intelligence affects economic decision making will be possible, including and understanding of the way in which different components of the process we outlined (including working memory and cognitive control) enter into the choice process.

\section{Acknowledgements}

The research was supported by a National Foundation grant SES 1357877 to AR.

\section{References and recommended reading}

Papers of particular interest have been highlighted as: * of special interest

[1] C. Spearman: General intelligence objectively determined and measured. The American Journal of Psychology 1904, 15: 201-293.

[2] Carroll JB: Human cognitive abilities: A survey of factor-analytic studies. Cambridge University Press; 1993.

[3] Bowles SB, Gintis H, Osborne, O: The Determinants of Earnings: Skills, Preferences, and Schooling. Journal of Economic Literature 2001, 39: 1137-1176. 
[4] Neal DA, Johnson WR: The Role of Premarket Factors in Black-White Wage Differences. Journal of Political Economy 1996, 104: 869--895.

[5] Gottfredson LS: Why $g$ Matters: The Complexity of Everyday Life. Intelligence 1997, 24: 79-132

[6] James JH, Stixrud J, Urzua S: The effects of cognitive and noncognitive abilities on labor market outcomes and social behavior. Journal of Labor Economics 2006, 24: 411-482.

[7] Garett J, Schneider WJ: IQ in the production function: evidence from immigrant earnings. Economic Inquiry 2010, 48:743-755.

[8] Shane F: On the ball: Cognitive reflection and decision making. Journal of Economic Perspectives 2005, 19:25-42.

[9] Burks SV, Carpenter JP, Goette, L, Rustichini, A: Cognitive skills affect economic preferences, strategic behavior and job attachment. Proceedings of the National Academy of Sciences 2009, 106:7745-7750.

[10] Oechssler JJ, Roider A, Schmitz PW: Cognitive abilities and behavioral biases. Journal of Economic Behavior and Organization 2009, 72: 147-152.

[11] Dohmen T, Falk A, Huffman D, Sunde U: Are risk aversion and impatience related to cognitive ability?. American Economic Review 2010, 100:1238-1260.

[12] Benjamin DJ, Brown SA, Shapiro JM: 2013. Who is behavioral? Cognitive ability and anomalous preferences. Journal of the European Economic Association 2013, 11: 1231-1255.

[13] Bernheim, BD: Rationalizable Strategic Behavior. Econometrica 1984, 52:1007-1028.

[14] Pearce, D: Rationalizable Strategic Behavior and the Problem of Perfection. Econometrica 1984, 52:1029-1050.

[15] Mischel W, Ebbesen EB, Raskoff Zeiss A: Cognitive and attentional mechanisms in delay of gratification. Journal of Personality and Social Psychology 1972, 21: 204-218.

[16] Chance MRA, Mead AP: Social behaviour and primate evolution. Symposium Society Experimental Biology 1953, 7:395--439.

[17] Jolly, A: Lemur social behavior and primate intelligence. Science 1966, 153: 501-506.

[18] Humphrey N: The social function of intellect. In Growing points in ethology. Edited by Bateson PG, Hinde RA, Cambridge University Press; 1976:303- 317.

[19] Dunbar RIM: The social brain hypothesis. Evolutionary Anthropology 1998, 6:178-190.

[20] Dunbar RIM, Shultz S: Understanding primate brain evolution. Philosophical Transactions of the Royal Soc. B. 2007, 362:649-658. 
[21] Costa-Gomes MA, Crawford V, Broseta B: Cognition and behavior in normal-form games: An experimental study. Econometrica 2001, 69: 1193-1235.

[22] Costa-Gomes MA, Crawford VP: Cognition and Behavior in Two-Person Guessing Games: An Experimental Study. American Economic Review 2006, 96: 1737-1768.

[23] Stahl DO, Wilson PW: On players' models of other players: Theory and experimental evidence. Games and Economic Behavior 1995, 10:218-254.

[24] Gill D, Prowse V: Cognitive Ability, Character Skills, and Learning to Play Equilibrium: A Level-k Analysis. Oxford Department of Economics Discussion Paper 712.

[25] Jones G: Are smarter groups more cooperative? Evidence from prisoner's dilemma experiments, 1959-2003. Journal of Economic Behavior and Organization 2008, 68: 489-497.

[26] Dal Bo, P: Cooperation under the Shadow of the Future: Experimental Evidence from Infinitely Repeated Games. American Economic Review 2005, 95:1591-1604.

[27*] Dal Bo P, Frechette GR: The Evolution of Cooperation in Infinitely Repeated Games: Experimental Evidence. American Economic Review 2011, 101: 411-429.

The paper tests the main game theoretic predictions on behavior of players in a repeated prisoner's dilemma, with a design along the lines described in the text; the discount factor in the theory corresponds to the probability that the game ends. The authors study cooperation in the Prisoner's Dilemma, and find that cooperation increases as the continuation probability increases, depending on the payoffs of the stage game, as the theory predicts.

[28] Chen C: Intelligence moderates reinforcement learning: a mini-review of the neural evidence. Journal of Neurophysiology 2014, 112:12-25.

[29] Diekhof EK, Kaps L, Falkai P, Gruber O. The role of the human ventral striatum and the medial orbitofrontal cortex in the representation of reward magnitude - an activation likelihood estimation meta-analysis of neuroimaging studies of passive reward expectancy and outcome processing. Neuropsychologia 2012, 50: 1252-1266.

[30*] Schlagenhauf F, Rapp MA, Huys QJ, Beck A, Wüstenberg T, Deserno L, Buchholz HG, Kalbitzer J, Buchert R, Bauer M, Kienast T, Cumming P, Plotkin M, Kumakura Y, Grace AA, Dolan RJ, Heinz A. Ventral striatal prediction error signaling is associated with dopamine synthesis capacity and fluid intelligence. Human Brain Mapping 2013, 34: 1490-1499.

In this study, subjects performed a reversal learning task, and had to choose between actions that could deliver a reward with high probability or a loss. The choice delivering the reward was periodically reversed as a function of reward history. The authors estimate the prediction error computed with a 
model-free learning model and used it as covariate for BOLD. They find a positive correlation between the prediction error in the ventral striatum (VS) and fluid intelligence.

[31*] van der Bos W, Crone EA, Guroglu B: Brain function during probabilistic learning in relation to IQ and level of education. Developmental Cognitive Neuroscience 2012, 2:S78-S89.

The task in this study was a simple probability learning task. The subjects had to choose between two options, giving a reward with high (correct choice) or low (wrong choice) probability. In this design, the optimal policy is to select the correct choice in every round irrespective of history. The subjects exhibited probability matching behavior (converging to the correct choice with a frequency equal to the probability of reward). The authors find a positive correlation between the difference between positive feedback when using the wrong rule and positive feedback when using the correct rule and IQ score (measured with WISC).

[32*] Hawes DR, DeYoung CG, Gray JR, Rustichini A. Intelligence moderates neural responses to monetary reward and punishment. Journal of Neurophysiology 2014, 111:1823-1832.

In this experiment subjects selected one of two choices against a pseudo-random sequence that gives the same sequence of gains and losses for all subjects. The authors find the decline in the BOLD signal after loss to be less steep in subjects with a higher IQ score.

[33] MacDonald PA, Ganjavi H, Collins DL, Evans AC, Karama S: Investigating the relation between striatal volume and IQ. Brain Imaging Behavior 2014, 8:52-9.

[34] Abernethy LJ, Cooke RWI, Foulder-Hughes L: Caudate and hippocampal volumes, intelligence, and motor impairment in 7-year-old children who were born preterm. Pediatric Research 2004, 55:884893.

[35*] Isaacs EB, Gadian DG, Sabatini S, Chong WK, Quinn BT, Fischl BR, Lucas A: The effect of early human diet on caudate volumes and IQ. Pediatric Research 2008, 63:308-314.

The study has two parts. The first illustrates the correlation between Verbal IQ and caudate volume. The second part reports a study where a group of subjects were randomly assigned to a post-natal highnutrient treatment: caudate volume and verbal IQ were significantly higher in the high-nutrient group. [36] Verstynen TD, Lynch B, Miller DL, Voss MW, Prakash RS, Chaddock L, Basak C, Szabo A, Olson EA, Wojcicki TR, Fanning J, Gothe NP, McAuley E, Kramer AF, Erickson KI (2012): Caudate nucleus volume mediates the link between cardiorespiratory fitness and cognitive flexibility in older adults. Journal of Aging Research 2012, 2012:1-11. 
[37] Burgaleta M, MacDonald PA, Martínez K, Román FJ, Álvarez-Linera J, González AR, Karama S, Colom R (2013): Subcortical regional morphology correlates with fluid and spatial intelligence. Human Brain Mapping 2013, 35:1957-1968.

[38] Grazioplene, RG, Ryman S, Gray, JR, Rustichini, A, Jung, RE, DeYoung, CG: Subcortical intelligence: Caudate volume predicts IQ in healthy adults. Human Brain Mapping 2015, 36:1407-1416.

[39] Börgers T, Morales AJ, Sarin R: Expedient and monotone learning rules. Econometrica 2004, 72 : 383-405.

[40] Busemeyer JR, Stout JC: A contribution of cognitive decision models to clinical assessment: Decomposing performance on the Bechara Gambling Task. Psychological Assessment 2002, 14:253262.

[41] March JG: Learning to be risk averse. Psychological Review 1996, 103:309-319.

[42] Sarin R, Vahid F: Payoff assessments without probabilities: A simple dynamic model of choice. Games and Economic Behavior 1999, 28:294-309.

[43] Denrell, J. Adaptive learning and risk taking. Psychological Review 2007, 114:177-187.

[44] Doll BB, Simon DA, Daw ND: The ubiquity of model-based reinforcement learning. Current Opinion in Neurobiology 2012, 22:1075-1081.

[45] Lee SW, Shimojo S, O'Doherty JP: Neural computations underlying arbitration between modelbased and model-free learning. Neuron 2014, 81:687-99.

[46] Daw ND, O'Doherty JP, Dayan P, Seymour B, Dolan RJ: Cortical substrates for exploratory decisions in humans. Nature 2006, 44:876-879. 